\title{
Biosynthesis of biodegradable polyhydroxyalkanotes biopolymers in genetically modified yeasts
}

\author{
${ }^{1 *}$ D. Abd-El-haleem; ${ }^{1}$ A. Amara; ${ }^{1}$ S. Zaki; ${ }^{2}$ A. Abulhamd; ${ }^{1}$ G. Abulreesh \\ ${ }^{1}$ Environmental Biotechnology Department and Protein Research Department, Genetic Engineering and Biotechnology \\ Research Institute (GEBRI), Mubarak City for Scientific Research and Technology Applications, New Burg-Elarab City, \\ Alexandria, Egypt \\ ${ }^{2}$ Microbiology Department, Faculty of Science, Alazhar University, Cairo, Egypt
}

Received 26 February 2007; revised 22 March 2007; accepted 18 July 2007; available online 1 September 2007

\begin{abstract}
In the recent decade, biosynthesis of the degradable biopolymers polyhydroxyalkanotes in transgenic yeasts became an important research task. Most research strategies depend on either metabolic engineering or molecular approaches. In the present work, research compared PHA biosynthesis in two types of yeasts; Saccharomyces cerevisiae and a non-convenient Kloeckera spp. Yeast strains were equipped in their cytoplasm with the phaABC$C_{R e}$ operon containing genes $p h b \mathrm{~A}, p h b \mathrm{~B}$ and $p h b \mathrm{C}$ of the PHA biosynthetic pathway of Ralstonia eutropha, which encode â-ketothiolase, NADPH-linked acetoacetyl-CoA reductase and PHA synthase, respectively. The transgenic strains Saccharomyces cerevisiae and Kloeckera sp. were able to produce PHA. The maximum content of the polymer detected in the recombinant strain INVSc1/PHA1 was $2.68 \%$ and only poly-3-hydroxybutyrate (PHB) accumulated. However, the non-conventional transgenic strain KY1/PHA was able to accumulate as maximum of $7.06 \%$ of the copolymer poly-(3-hydroxybutyrateco-poly-3-hydroxyvalerate) (PHV). Western blot analysis confirmed expression of the phaABC $C_{\mathrm{Re}}$ operon in the transgenic yeast strains. The nature of the PHA thus produced by all tested strains was analyzed by ${ }^{1} \mathrm{H}$ and ${ }^{13} \mathrm{C}$ nuclear magnetic resonance (NMR) spectroscopy.
\end{abstract}

Key words: Recombinant yeasts, polyhydroxyalkanotes, poly-3-hydroxybutyrate, nuclear magnetic resonance

\section{INTRODUCTION}

A major problem of waste and environmental pollution is that plastics produced by the petrochemical industry are not biodegradable and therefore accumulate in the environment (Juang and Chiou, 2007; Torabian, et al., 2004). However, some microbial species have been identified which produce "environmentally friendly" biodegradable plastics, in particular polyesters of the poly-hydroxyalkanoates (PHA) family. Polyhydroxyalkanotes (PHAs) are structurally simple macromolecules that accumulate as discrete granules to levels as high as $90 \%$ of the cell dry weight. They are generally believed to play a role as sink for carbon and reducing equivalents (Senior, et al., 1972). Poly(3-hydroxybutyrate) $\mathrm{P}(3 \mathrm{HB})$ is the archetype of PHAs and was described first as an important bacterial product by Lemoigne (1923) . The bacterial origins of PHA make these polyesters a natural material, and therefore many microorganisms have the ability to degrade these macromolecules (Jendrossek, et al., 1996). It is now well recognized that $\mathrm{P}$ (3HB) is

*Corresponding Author Email: abdelhaleemm@yahoo.de Tel.: +974 485 2702; Fax: +974 835061 accumulated as a common carbon energy storage compound in Gram-negative and Gram-positive bacteria as well as archaea (Fernandez-Castillo, et al., 1986 and Steinbüchel and Füchtenbusch, 1998). The major commercial drawback of the bacterial PHAs is their high production cost, making them substantially more expensive than synthetic plastics (Poirier, et al., 1995). Therefore, engineering of novel pathways in eukaryotic cell systems seems to be a beneficial alternative to the production of PHAs in bacteria. Yeast cells can be used as models to gain information on PHAs synthesis in eukaryotes (Leaf, et al., 1996, Carlson, et al., 2002, Breuer, et al., 2002 and Terentiev, et al., 2004). Seebach, et al., (1994) describes evidence of PHB existing in yeast and many other eukaryotic cells, which contain small amounts of low molecular mass PHBs. Here, the PHBs function as complexes with polyphosphate in membrane transport. In addition, yeasts as hosts for synthesis of PHAs have certain advantages over bacteria. First, yeasts have been studied intensively from physiology, molecular biology and biotechnology points of 
views. Second, yeasts are physiologically flexible and they are larger than bacteria (Carlson, et al. 2002 and Terentiev, et al., 2004). Moreover, yeast like, Saccharomyces cerevisiae, Klyveromyces marxianus, Candida utilis and others, has been approved as a GRAS microorganism by Food and Drug Administration (Boze, et al., 1994). Expression of the Ralstonia eutropha $\mathrm{P}(3 \mathrm{HB})$ biosynthetic pathway was achieved successfully in the yeast Saccharomyces cerevisiae by (Leaf, et al., 1996) who reported that the expression of PHB synthase in the yeast cytoplasm is sufficient for PHB accumulation and, wild type yeast synthesizes D-3HB-CoA, which could arise from intermediates in fatty acid synthesis or through $\beta$-oxidation. Three thiolases are known to exist in $S$. cerevisiae functioning in mitochdria, cytoplasm and peroxisomes (Igual, et al., 1992). Recently, Terentiv et al. (2004) reported that although PHA synthesis has been demonstrated in the nonconventional yeast $A$. adeninivorans, their results suggest that many aspects of PHA synthesis, metabolism and regulation need to be explain before the organism that can be used for the synthesis of PHA on a commercial scale. Therefore, in the present work, we compared the expression of the genes encoding the polyhydroxybutyrate (PHB) biosynthetic pathway in Ralstonia eutropha (3ketothiolase, phaA or bktB; acetoacetyl-CoA reductase, phaB; and PHA synthase, phaC) in the convenient yeast Saccharomyces cerevisiae and the non-convenient yeast isolate Kloeckera spp. KY1.

\section{MATERIALS AND METHODS}

Strains and culture conditions

Plasmids were maintained and propagated in Escherichia coli DH5-alpha according to Sambrook, et al., (1989). S. cerevisiae diploid strain INVSc1 (his3D1 leu2 trp1-289 ura3-52) was obtained from Invitrogen (Groningen, The Netherlands). The wild type yeast Kloeckera spp. strain KY1, isolated from molasses collected from El-haoamdeya sugarcane factory, Giza, Egypt was identified biochemically with the API20C Aux system (BioMerieux Vitek, Inc., Hazelwood, Mo.) with very good identification. Transgenic yeasts harboring the PHB synthesis genes were maintained in leucine-deficient media $(0.67 \%$ yeast nitrogen base without amino acids [Difco, Detroit, Mich.], $0.5 \%$ ammonium sulfate, $2 \%$ glucose, and $0.4 \mathrm{~g}$ of leucine dropout supplement (Clontech, Palo Alto, Calif).

\section{Construction of an episomal vector}

A cassette including the three genes of the PHA biosynthesis pathway of Ralstonia eutropha \{betaketothiolases (phbA); NADPH-linked-acetoacetyl-CoA reductase $(p h b B)$ and PHA synthase $(p h b C)\}$ was obtained from the plasmid pBHR68 (pBluscriptSK') that contains a $5.2 \mathrm{~Kb}$ fragment from chromosomal DNA of Ralstonia eutropha (Spiekermann, et al., 1999). Subsequently, the cassette was digested with endonucleases enzymes Xbal and BamHI and subcloned by ligation into the pBK-CMV phagemid vector (Stratagene, La Jolla, CA, USA). The resulting construct designated $\mathrm{pBK} / \mathrm{PHA}$ was transferred to both INVSc1 and KY1 cells by transformation using the lithium acetatemethod (Gietz, et al., 1995). Transformants were recovered on leucine deficient medium. To screen for presence/absence of $\mathrm{PHB}$, transformants were grown on leucine deficient agar plates containing $25 \mu \mathrm{g} / \mathrm{L}$ NileRed (PHB stain) and incubated at $30{ }^{\circ} \mathrm{C}$ for $72 \mathrm{~h}$. Thereafter, plates were exposed to ultraviolet light $(300$ $\mathrm{nm}$ ) and illuminated clones were recorded as positives.

\section{Western blot analysis}

Protein concentrations were determined by the method of Bradford (Bradford, 1976), using bovine serum albumin as a standard. Western blotting was performed using the Semi-dry Fastblot apparatus (Biometra, Germany). Protein was detected applying the respective monospecific, polyclonal anti-PhaC antiserum and alkaline-phosphatase-antibody conjugate as second antibody. The SDS-PAGE gel, Whatman paper and membrane were all incubated in transfer buffer for $10 \mathrm{~min}$ and afterwards put into the semi-dry transfer apparatus according to this sequence: anode, Whatman paper, membrane, SDS-PAGE gel, Whatman paper and cathode (Kyhse-Andersen, 1984). The chromogenic visualization of the bound antibodies was done by immersing the membrane in a $10 \mathrm{~mL}$ solution of 5-bromo4-chloro-3-indolyl phosphate for 10-30 min until the reaction complex was visualized clearly. The color reaction was stopped by rinsing the membrane with distilled water.

\section{PHB production}

For PHB production, a stationary-phase culture grown in leucine-deficient media was harvested by centrifugation and cells were washed once in water and resuspended in mineral salts medium (MSM) (Schlegel et al., 1961) supplemented with $0.1 \%$ glucose, $0.5 \%$ of 
the detergent Tween 60 (Sigma, St. Louis, Mo.), and $0.1 \%$ fatty acid (oleic acid). Cells were grown at $30{ }^{\circ} \mathrm{C}$ for an additional 1 to 6 days before harvest of the cells for PHB analysis. The $\mathrm{pH}$ of the growth media was 6.0.

PHB extraction procedure was performed according to Findlay and White (Findlay and White, 1983). Lyophilized yeast cell sediments were placed in a Soxhlet extractor lined with glass wool and wrapped with a Yeast strains resistance strip heater. Enough chloroform to cover the sample was added, and the sample was sonicated for $10 \mathrm{~min}$. The sample was extracted overnight in a total of $125 \mathrm{~mL}$ of chloroform. The extraction thimble of the Soxhlet extractor was heated so that the chloroform present boiled, maintaining solubility of the polymers. The chloroform was recovered and removed in a rotary evaporator in vacuum. Subsequently, the polymer was redissolved in hot chloroform and PHA was recovered from the chloroform by nonsolvent precipitation and filtration. Methanol was used as the nonsolvent (4-6 volumes). To determine PHB yield, the correlation between production of PHB and dry cell weight was determined by Spearman's test (Conver, 1971).

\section{PHB analysis}

The NMR spectra were recorded on a JOEL ECA 500 spectrometer. The $500 \mathrm{MHz}{ }^{1} \mathrm{H}-\mathrm{NMR}$ spectra were recorded from a $\mathrm{CDCl}_{3}$ solution of the $\mathrm{PHA}(30 \mathrm{mg} / \mathrm{mL})$ at $20^{\circ} \mathrm{C}, 1.30809856 \mathrm{~s}$ acquisition times and 12.5250501 $\mathrm{KHz}$ spectral width. The ${ }^{13} \mathrm{C}-\mathrm{NMR}$ spectra were recorded from a $\mathrm{CDCl}_{3}$ solution of the samples using ${ }^{1} \mathrm{H}$ decoupling, $0.69206016 \mathrm{~s}$ acquisition time and 47.348485
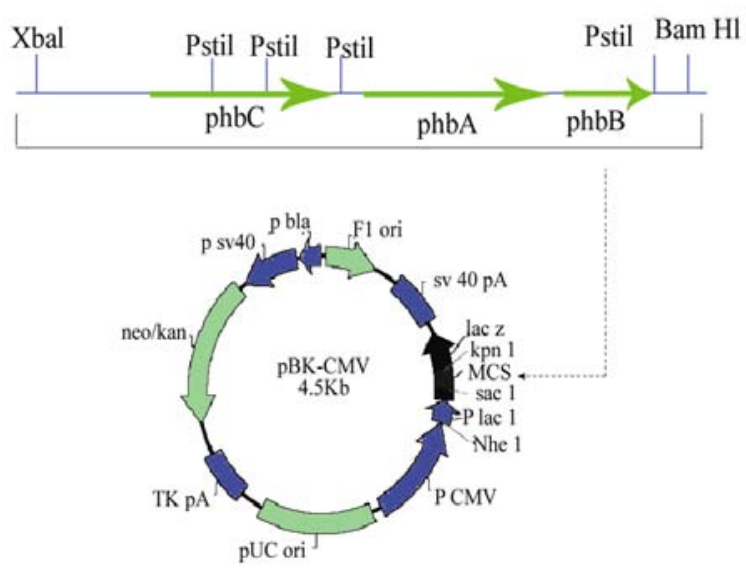

Fig 1: Diagram of the episomal vector pBK-CMV, details of construction are given in the text. The linear map represents the PHB operon of R. eutropha derived from plasmid pBHR68
KHz spectral width. ${ }^{1} \mathrm{H}-\mathrm{NMR}$ and ${ }^{13} \mathrm{C}$-NMR chemical shifts were referred to $\mathrm{CHCl}_{3}$ and $\mathrm{CDCl}_{3}(\mathrm{c}=7.24 \mathrm{ppm}$ and $\mathrm{c}=77.0 \mathrm{ppm}$, respectively).

\section{RESULTS}

Plasmid construction and phb expression

The yeast plasmid pBK-CMV (Stratagene, La Jolla, CA, USA), carrying the PHB biosynthesis genes $p h b A$, $p h b B$ and phbC of Ralstonia eutropha is presented (Fig. 1). Saccharomyces cerevisiae INVSc1 and Kloeckera spp KY1 were transformed with the constructed plasmid designated $\mathrm{pBK} / \mathrm{PHB}$. Yeast colonies containing plasmid $\mathrm{pBK} / \mathrm{PHB}$ were then screened for PHB expression using the Nile-Red staining assay described by Spiekermann et al., (1999). To confirm results of the Nile-Red assay, positive clones were screened for the presence of inserts by restriction mapping (Sambrook, et al., 1989). Moreover, PCR detection of PHB genes in the positive yeast clones were performed using PHA specific PCR primers and protocols described elsewhere (Sheu et al., 2000). Based on screening results the transgenic yeasts designated S. cerevisiae INVSc1/PHA and Kloeckera spp KY1/PHA were chosen for further characterization and analysis.

\section{Western blot analysis}

Recombinant yeasts INVSc1/PHA1, KY1/PHA containing plasmid $\mathrm{pBK} / \mathrm{PHB}$ and their native strainsINVSc1 and KY1 were tested for expression of the PHA synthase by Western blot analysis using the specific anti-serum toward the C-terminal oligopeptide of $P h b C_{\mathrm{Re}}$ (Fig. 2). Yeast cells were grown to the stationary phase in leucine-deficient broth medium supplemented with glucose, oleic acid, and the detergent Tween 60 . Tween is a detergent containing oleic acid that is esterified to the polyoxyeth-ylenesorbitan backbone. This detergent was used to insure solubilization of the free oleic acid added to the medium. Resolution of crude cell extracts (Fig. 2) from the transgenic yeasts INVSc1/ PHA1, KY1/PHA and the wild type strain KY1 identified protein bands at approximately $66 \mathrm{kDa}$. However, protein of equivalent size to $P h b C_{\mathrm{Re}}$ protein did not appear in cell extracts from the plasmid free host strain INVSc1 indicating absence of active PhaC enzyme.

\section{PHA accumulation}

Transgenic yeast strains INVSc1/PHA1 and KY1/PHA containing plasmid $\mathrm{pBK} / \mathrm{PHA}$ and their native strains were tested for production of PHB. Cells were grown in $250 \mathrm{~mL}$ Erlenmeyer flasks containing $50 \mathrm{~mL}$ MSM medium at 
$30^{\circ} \mathrm{C}$ on a rotary shaker at $200-\mathrm{rpm} / \mathrm{min}$. All flasks were exposed to the same conditions, and three separate flasks were inoculated from the same culture in each case. Data from shake flasks (Table 1) indicated that plasmid-free cells of strain INVSc1 did not contain measurable PHB. In contrast, both plasmid-containing strains INVSc1/PHA1 and KY1/PHA and the wild type strain KY1 grown under the same conditions contained PHA (\% of the cell dry weight) in the order of $7.06 \%$, $2.68 \%$ and $0.83 \%$. As shown in Table 1, PHB production in transgenic yeasts INVSc1/PHA1, KY1/PHA and the wild type strain KY1 increased when the incubation period increased until it reached a maximum at day 4 (96 h). However, the lowest PHB productivity was observed after $24 \mathrm{~h}$ incubation with strain INVSc1/PHA1 and after $144 \mathrm{~h}$ incubation with KY1/PHA and its native strain KY1.

\section{PHA analysis}

Both ${ }^{1} \mathrm{H}$ and ${ }^{13} \mathrm{C}$ NMR spectra analyses of the purified PHA polymer from the recombinant yeast strain KY1/ PHA revealed production of the copolymer poly (3hydroxybutyrate-co-3-hydroxyvalerate) (PHBV). As shown in Fig. 3, the ${ }^{1} \mathrm{H}$ NMR spectrum of the copolymer has the characteristic signals of $\mathrm{HB}$ and $\mathrm{HV}$ units: ä ppm: 0.9, $\mathrm{t}\left(\mathrm{CH}_{3}-5\right), 1.3, \mathrm{~d}\left(\mathrm{CH}_{3}-9\right), 1.6, \mathrm{~m}\left(\mathrm{CH}_{2}-4\right), 2.58, \mathrm{~m}$ $\left(\mathrm{CH}_{2}-2\right.$ and $\left.\mathrm{CH}_{2}-7\right)$, and 5.24, $\mathrm{m}(\mathrm{CH}-8$ and $\mathrm{CH}-3)$. The ${ }^{13} \mathrm{C}$ NMR analysis supported the results from the proton NMR analysis. The resonance of the monomers at 19.87 (9), 40.80 (7), 67.64 (8), and 169.26(1) ppm were assigned by data comparison to $3 \mathrm{HB}$ according to the previous report (Doi et al., 1986), whereas the resonances at 22.10 (4), 38.65 (2) and $71.91 \mathrm{ppm}$ were referred to $3 \mathrm{HV}$. In contrast, ${ }^{1} \mathrm{H}$ NMR spectral analyses of the purified polymer from the recombinant yeast strain INVSc1/ PHA1 revealed the presence of 3,2 and 1 protons at chemical shifts 1.2, 2.4-2.6 and 5.3, respectively (Fig. 4). The molecular composition of the polyester as indicated by chemical shifts, generates a structure of $\left(\mathrm{CH}_{2}-\mathrm{CH}\right)$ backbone and assigned the presence of $\left(\mathrm{CH}_{3}\right)$

Table 1: The yield of PHA produced in the recombinant and wild type yeast strains

\begin{tabular}{cccc}
\hline $\begin{array}{c}\text { Incubation period } \\
(\mathrm{h})\end{array}$ & \multicolumn{3}{c}{ PHB \% of cell dry weight } \\
\hline 24 & 0.22 & 1.16 & 0.17 \\
48 & 1.15 & 2.93 & 0.76 \\
72 & 2.68 & 7.06 & 0.83 \\
96 & 1.67 & 3.58 & 0.48 \\
120 & 1.19 & 1.41 & 0.53 \\
144 & 0.44 & 0.45 & 0.08 \\
\hline
\end{tabular}

group. The ${ }^{13} \mathrm{C}$ NMR spectrum (at $500 \mathrm{MHz}$ ) of the polymer on the other hand showed chemical shifts at $19.87,40.86,67.70$ and $169.26 \mathrm{ppm}$, which assigned the presence of $\left(\mathrm{CH}_{3}\right),\left(\mathrm{CH}_{2}\right),(\mathrm{CH})$ and $(\mathrm{C}=\mathrm{O})$ groups respectively (Fig. 4). As shown in Fig. 5, the ${ }^{1} \mathrm{H}$ and ${ }^{13} \mathrm{C}$ NMR spectra of the PHA polymer produced by the wild type yeast strain KY1 have some characteristic signals of the copolymer PHBV. The ${ }^{1} \mathrm{H}$ NMR revealed the presence of $\mathrm{HB}(\mathrm{B})$ and HV (V) units: ä ppm: 1.27 (d, J - 6.21; H-4B), 0.83 (t, J = 7.34; H-5V), while ${ }^{13} \mathrm{C}$ NMR indicate the presence of signals: $19.74(\mathrm{C}-4 \mathrm{~B})$; $26.76(\mathrm{C}-4 \mathrm{~V})$ and $38.65(\mathrm{C}-2 \mathrm{~V})$, respectively.

\section{DISCUSSION AND CONCLUSION}

Previously, Leaf et al., (1996), Breuer, et al., (2002) and Terentiev et al., (2004) reported that no increase in PHA levels produced by expressing the entire threegene PHA pathway compared with those found in yeast transformants where only the $p h b C$ gene was expressed. They suggested that competition for the precursors from pathways may lead to the synthesis of other storage compounds. If so, competing pathways must be elucidated and ways to reduce them must be developed in order to increase the polymer yield. In the present work, the vector pBK-CMV (Stratagene) equipped with a $5.2 \mathrm{~Kb}$ fragment from chromosomal DNA of Ralstonia eutropha including the three genes of the PHA biosynthesis pathway of Ralstonia eutropha was used as a shuttle expression vector. According to that described in Stratagene manual (Catalog \# 212209), the vector pBK-CMV was derived from a high-copy-number pUC-based plasmid and allows expression in both eukaryotic and prokaryotic systems.

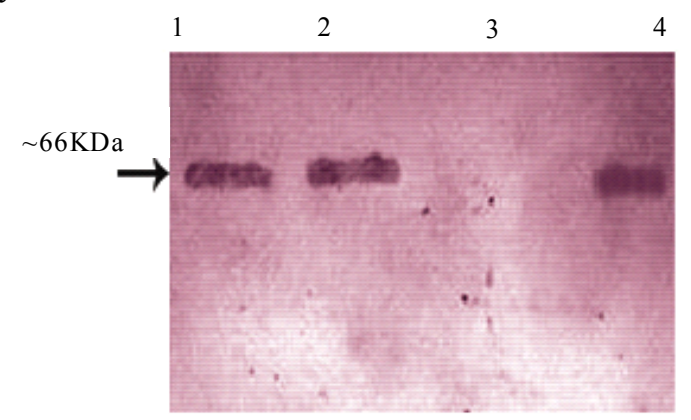

Fig. 2: Detection of $P h a C$ polymerase by Western blot analysis of the recombinant strains INVSc1/PHA1 (Lane 1), KY1/PHA (Lane 2), free plasmid strain S. cerevisiae INV (Lane 3) and the wild type strain KY1 (Lane 4). Western blotting was performed with polyclonal antibodies raised against $\mathrm{PhaCl}$ of $R$. eutropha. All lanes contained $25 \mu \mathrm{g}$ of protein 
Eukaryotic expression is driven by the cytomegalovirus (CMV) immediate early promoter in the pBK-CMV phagemid vector. Stable clone selection in eukaryotic cells is made possible with G418 by the presence of the neomycin- and kanamycin-resistance gene, which is driven by the SV40 early promoter with thymidine kinase (TK) transcription termination and polyadenylation signals.

In the pBK-CMV phagemid vector, prokaryotic expression is driven by the lac promoter, which is repressed in the presence of the LacI protein and is inducible by IPTG. Positive yeast colonies containing the designated plasmid $\mathrm{pBK} / \mathrm{PHB}$ were screened for PHB expression using the Nile-Red staining assay (Spiekermann, et al., 1999). It is known that the Nile-red stain emitted strongly positive red fluorescence signals only with hydrophobic compounds like PHAs and lipids. Nile-red intended to show any lipid particles inside the cells and it did not react with any tissue constituent except by solution and could be detected by fluorescence spectroscopy or flow cytometery (Terentiev, et al., 2004). The results of the Nile-Red staining assay were compatible with both restriction mapping and the PCR detection assays of PHB genes. The band intensities in the Western blot assay of the wild type strain KY1 were equivalent when comparing the transgenic yeasts INVSc1/PHA1 and KY1/PHA. This agrees with the results reported early by Leaf, et al., (1996). Thus, this led us to conclude that enzyme activities other than the synthase were limiting PHA production in yeasts. Spectroscopic analysis of the PHA polymer extracted from the recombinant yeast strain KY1/PHA revealed production of the copolymer poly (3hydroxybutyrate-co-3-hydroxyvalerate) (PHBV).
Copolymers such as poly (3-hydroxybutyrate-co-3hydroxyvalerate) (PHBV) are more flexible than the homopolymer PHB because of reduced crystallinity and are suitable for many commercial applications. PHBV has been produced commercially by growing Ralstonia eutropha on glucose and propionate (Byrom, 1992 and Holmes et al., 1984) but the costs of this process prohibits large-scale fermentations. Production of PHAs by means of genetic engineering of yeasts is expected to reduce costs to economical levels and production of PHB homopolymer in yeast has been demonstrated (Leaf et al., 1996). Here we through this work report the metabolic engineering of a recombinant but non-conventional yeast strain KY1/PHA able to produce PHBV copolymer. Recently, Terentiev et al., (2004) detected both PHB and PHV polymers in recombinant non-conventional yeast Arxula adeninivorans equipped with the genes $p h b A$, phbB and $p h b C$ of the polyhydroxyalkanotes (PHA) biosynthetic pathway of Ralstonia eutropha. In contrast, the characteristic signals for other hydroxyalkanoaic acids, however, were totally lacking and confirmed the homopolymeric nature of the compound isolated from the transgenic yeast INVSc1/ PHA1. The absence of detectable amounts of 3-HV in strain INVSc1/PHA1 is most likely the result of insufficient flux of substrates to synthesize 3-ketovaleryl-CoA for production of PHBV. Our results suggest that additional metabolic modification to boost the levels of propionyl substrate in the yeast strain INVSc1/PHA1 is required for efficient synthesis of PHBV. Spectra analysis of the PHA polymer produced by the wild type yeast strain KY1 has some characteristic signals of the copolymer PHBV.

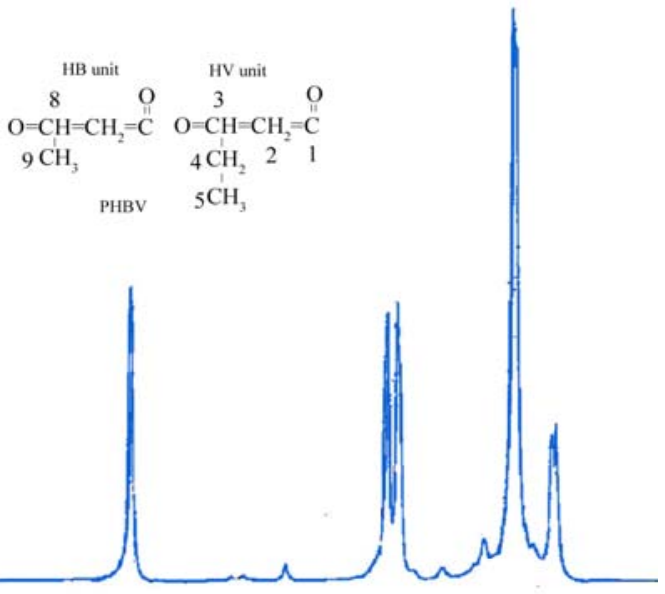

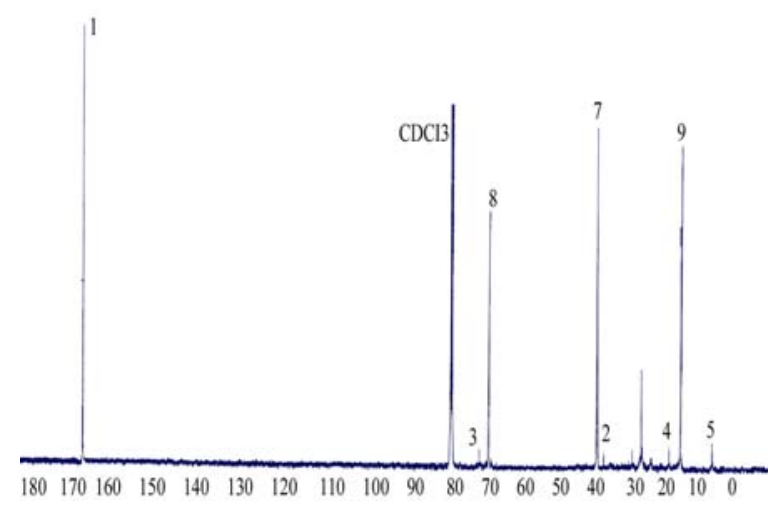

Fig. 3: ${ }^{1} \mathrm{H}$ and ${ }^{13} \mathrm{C}$ NMR spectra analysis of the polymer produced by the non-conventional recombinant yeast strain KY1/PHA 
PHAs in wild type yeast were originally reported by Seebach et al., (1992 and 1994) and Leaf et al., (1996) followed by Safak et al., (2002). However, the authors have not attempted monomer detection by ${ }^{1} \mathrm{H}$ and $/$ or ${ }^{13} \mathrm{C}$ NMR, though such species may be relatively insoluble in chloroform. Seebach et al., (1994) suggests that native low molecular mass PHB may be complexes with various lipids or proteins, making its solubility behavior unpredictable.

In conclusion, the recombinant Saccharomyces cerevisiae strain INVSc1/PHA1 produced $2.68 \% \mathrm{PHB}$ of the cell dry weight. Previously, cells of Saccharomyces cerevisiae transformed with the PHA synthase plasmid accumulated up to $0.5 \%$ of cell dry weight as PHA Leaf et al., (1996). The maximum amount of PHA accumulated in recombinant $S$. cerevisiae growing in media containing fatty acids was $0.45 \%$ of the dry weight (Poirier et al., 2001). Similarly, the maximum amount of PHB found in $S$. cerevisiae growing on glucose was $6.7 \%$ (Breuer, et al.,

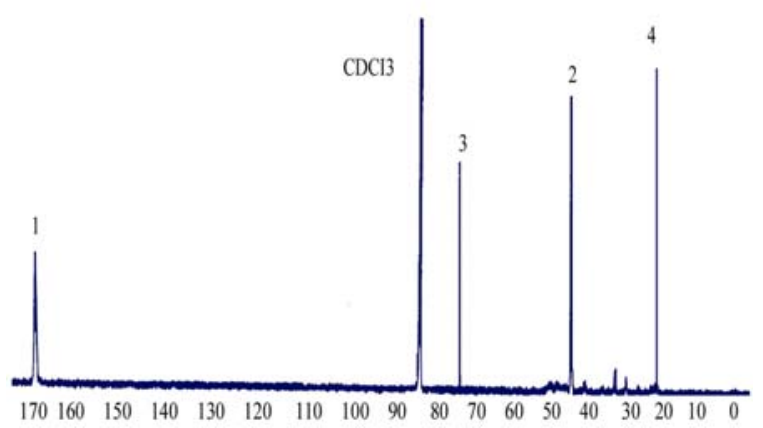

2002). Out of this survey, it is possible to conclude that Saccharomyces cerevisiae is suitable for genetic manipulation and, therefore, for the synthesis of PHB. However, the rates of synthesis are very low. Obviously, it is undesirable when the genes are episomally located (Terentiev, et al., 2004). For these reasons, to achieve a high mitotic stability of PHA production in genetically modified yeast, the use of different hosts with more suitable metabolic characteristics may be advantageous. In this regard, the results achieved by Terentiev, et al., (2004) using the non-conventional but transgenic yeast Arxula adeninivorans revealed accumulation of a maximum amount of $0.107 \% \mathrm{PHB}$ and $0.128 \% \mathrm{PHV}$, respectively. In contrast, our constructed nonconventional recombinant yeast Kloeckera spp. strain KY1/PHA was able to accumulate PHBV up to $7.06 \%$. Therefore and due to the wide commercial applicability of PHBV, the results obtained suggest that many aspects of its synthesis, metabolism and regulation need to be

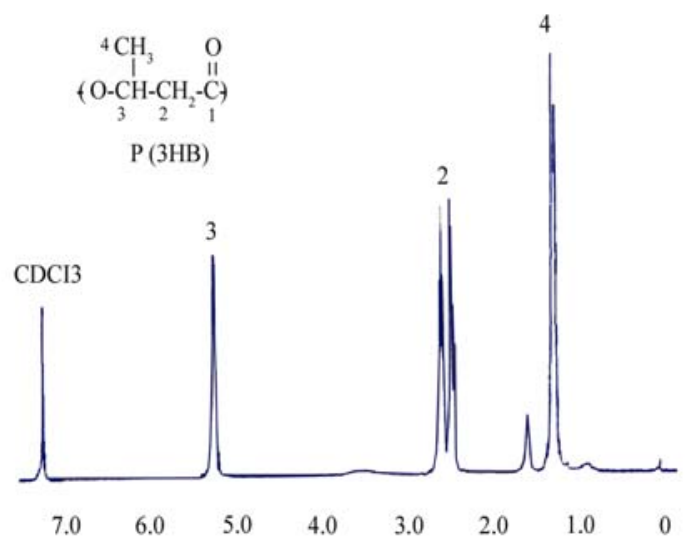

Fig 4: ${ }^{1} \mathrm{H}$ and ${ }^{13} \mathrm{C}$ NMR analysis of the PHA polymer produced by the recombinant strain INVSc1/PHA1

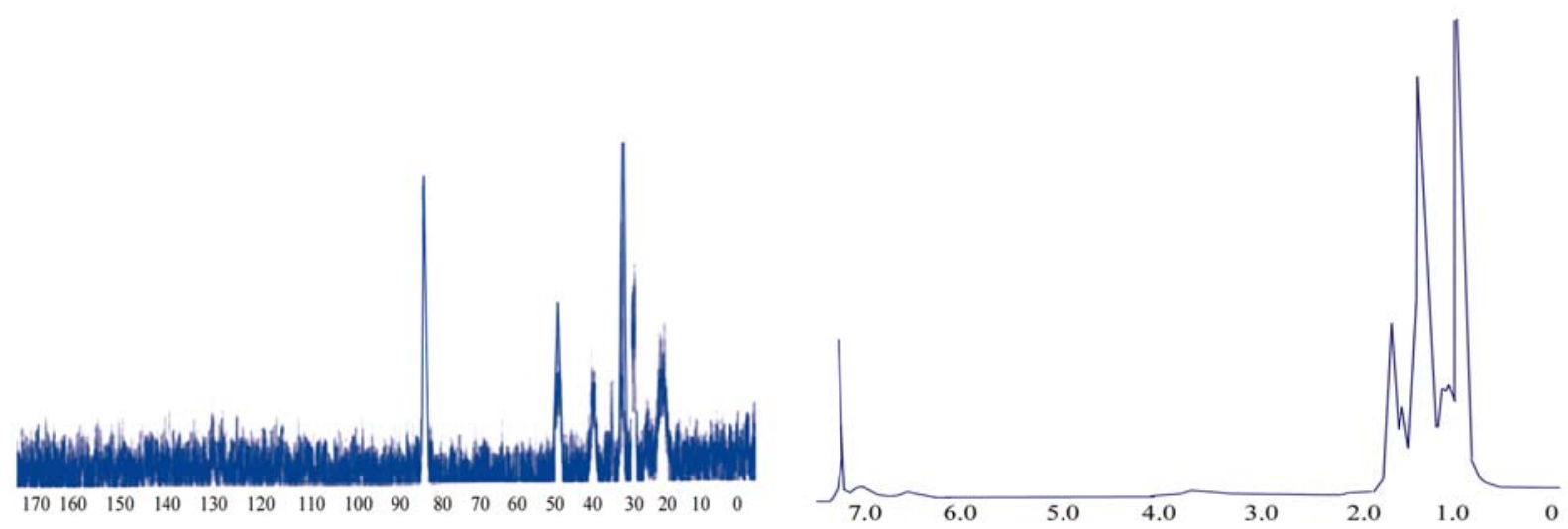

Fig. 5: ${ }^{1} \mathrm{H}$ and ${ }^{13} \mathrm{C}$ NMR analysis of the PHA polymer produced by the wild type yeast strain $\mathrm{KY} 1$ 
elucidated before the organism can be used for the synthesis of this polymer on a commercial scale.

\section{ACKNOWLEDGEMENTS}

This work was carried out under the program of the Egyptian Strategy for Biotechnology and Genetic Engineering contract No. 83. The program is administrated by the Science and Technology Center at the Academy of Scientific Research and Technology. Great thanks to Professor Malcolm Potts the lead scholar at Biological Sciences Department, College of Arts and Sciences, Qatar University for his reviewing and valuable notes of this paper.

\section{REFERENCES}

Boze, H.; Moulin, G.; Galzy, P., (1994). Production of food and fodder yeast., Crit. Rev. Biotechnol., 12, 5-86.

Bradford, M.M., (1976). A rapid and sensitive method for the quantitation of microgram quantities of protein utilizing the principle of protein-dye binding., Anal. Biochem., 72, 248254.

Breuer, U.; Terentiev, Y.; Kunze, G.; Babel, W., (2002). Yeasts as Producers of Polyhydroxyalkanoates: Genetic Engineering of Saccharomyces cerevisiae., Macromol. Biosci., 2, 380-386.

Byrom, D., (1992). Production of poly-hydroxybutyrate:polyhydroxyvalerate copolymers., FEMS Microbiol. Rev., 102, 247-250.

Carlson, R.; Fell, D.; Srienc, F., (2002). Metabolic Pathway analysis of recombinant yeast for rational strain development., Biotechnol. Bioeng., 79, 121-134.

Conver, W.J., (1971). Practical non parametric statics, John Weleyard and Sonc. Inc, New York.

Doi, Y., Kunioka, M.; Nakamura, Y.; Soga, K., (1986). Nuclear magnetic resonance studies on poly(L-hydroxybutyrate) and a copolyester of L-hydroxybutyrate and L-hydroxyvalerate isolated from Alcaligenes eutrophus H16., Macromolecules., 19, 2860-2864

Fernandez-Castillo, R.; Rodrigues-Valera, F.; Gonzalez-Ramos, J.; Ruiz-Berraquero, F., (1986). Accumulation of poly(ßhydoxybutyrate) by halobacteria., Appl. Environ. Microbiol., 51, 214-216.

Findlay, R.H.; White, D.C., (1983). Polymeric betahydroxyalkanoates from environmental samples and Bacillus megaterium., Appl. Environ. Microbiol., 45, 71-78.

Gietz, R.D.; Schiestl, R.H.; Willems, A.R.; Woods, R.A., (1995). Studies on the transformation of intact yeast cells by the LiAc/ SS-DNA/PEG procedure., Yeast., 11, 355-360.

Holmes, P.A.; Collins, S.H.; Wright, F., (1984). 3-hydroxybutyrate polymers., US Patent. 4, 477-654.

Igual, J.C.; Gonzalez-Bosch, C.; Perez-Ortin, J.E., (1992). The POTL gene for yeast peroxisomal thiolase is subject to there different mechanisms of regulation., Mol. Microbiol., 6, 1867-1875.

Jendrossek, D.; Schirmer, A.; Schlegel, H.G., (1996). Biodegradation of polyhydroxyalkanoic acids., Appl. Microbiol. Biotechnol., 46, 451-463.

Juang, D.F.; Chiou, L.J., (2007). Microbial population structures in activated sludge before and after the application of synthetic polymer. Int. J. Environ. Sci. Tech., 4 (1), 119-125.
Kyhse-Andersen, J., (1984). Electroblotting of multiple gels: a simple apparatus without buffer tank for rapid transfer of proteins from polyacrylamide to nitrocellulose., J. Biochem. Biophys. Methods., 10, 203-209.

Leaf, T.A.; Peterson, M.S.; Stoup, S.K.; Somers D.; Srienc, F., (1996). Saccharomyces cerevisiae expressing bacterial polyhydroxybutyrate synthase produces poly-3hydroxybutyrate., Microbiology., 142, 1169-1180.

Lemoigne, M., (1923). Production d'acide â-oxybutyrique par certaines bact'eries du groupe du Bacillus subtilis., C.R. Acad. Sci., 176, 1761-1765.

Poirier, Y.; Erard, N.; Macdonald-Comber, J., (2001). Synthesis of polyhydroxyalkanoate in the peroxisome of Saccharomyces cerevisiae using intermediates of fatty acid oxidation., Appl. Environ. Microbiol., 67, 5254-5260.

Poirier, Y.; Nawrath, C.; Somerville C., (1995). Production of polyhydroxyalkanoates, a family of biodegradable plastics and elastomers, in bacteria and plants., Biotechnology., 13, 142-150.

Safak, S.; Mercan, N.; Aslim, B.; Beyatli, Y., (2002). A study on the production of Poly-Beta-Hydroxybutyrate by some eukyryotic microorganisms., Tr. El. J. Biotechnol., 11-17.

Sambrook, J.; Fritsch, E.F.; Maniatis, T., (1989). Molecular cloning: a laboratory manual, $2^{\text {nd. }}$ Ed. Cold Spring Harbor Laboratory Press, Cold Spring Harbor, N.Y.

Schlegel, H.G.; Kaltwasser, H.; Gottschalk, G., (1961). Ein Submersverfahren zur Kultur wasserstofoxidierender Bakterien: Wachstumsphysiologische Untersuchungen., Arch. Microbiol., 38, 209-222.

Seebach, D.; Brunner, A.; Burger, H.M.; Schneider, J.; Reusch, R.N., (1994). Isolation and ${ }^{1} \mathrm{H}-\mathrm{NMR}$ spectroscopic identification of poly (3-hydroxybutanoate) from prokaryotic and eukaryotic organisms, Determination of the absolute configuration $(R)$ of the monomeric unit 3hydroxybutanoic acid from Escherichia coli and spinach., Eur. J. Biochem., 224, 317-328.

Seebach, D.; Beck, A.K.; Breitschuh, R.; Job, K., (1992). Direct degradation of the biopolymer poly $[(R)-3$-hydroxybutyric acid] to $(R)$-3-hydroxybutanoic acid and its methyl ester., Org. Synth., 71: 39-47.

Senior, P.J.; Beech, G.A.; Ritchie, G.A.F.; Dawes, E.A., (1972). The role of oxygen limitation in the formation of poly-âhydroxybutyrate during batch and continuous culture of Azotobacter beijerinckii., Biochem. J., 128, 1193-1201.

Sheu, D.S.; Wang, Y.T.; Lee, C.Y., (2000). Rapid detection of polyhydroxyalkanoates-accumulating bacteria isolated from the environment by colony PCR., Microbiology, 146, 2019-2025.

Spiekermann, P.; Reham, B.H.; Klascheuer, R.; Baumeister, D.; Steinbüchel, A., (1999). A sensitive, viable-colony staining method using Nile red for direct screening of bacteria that accumulate polyhydroxyalkanolic acids and other lipid storage compounds., Arch. Microbial., 171, 73-80.

Steinbüchel, A.; Füchtenbusch, B., (1998). Bacterial and other biological systems for polyester production., Trends Biotechnol., 16, 419-427.

Terentiev, Y.; Breuer, U.; Babel, W.; Kunze, G., (2004). Nonconventional yeasts as producers of polyhydroxyalkanoates genetic engineering of Arxula adeninivorans., Appl. Microbiol. Biotechnol., 64, 376-381.

Torabian, A.; Hassani, A.; Moshirvaziri H.S., (2004). Physicochemical and biological treatability studies of urban solid waste leachate. Int. J. Environ. Sci. Tech., 1 (2), 103-107. 


\section{AUTHOR (S) BIOSKETCHES}

Abd-El-Haleem, D., is an associate professor in Environmental Biotechnology Department, Genetic Engineering and Biotechnology Research Institute, Mubarak City for Scientific Research and Technology Applications, Alexandria, Egypt and Biological Sciences Department, College of Arts and Sciences, Qatar University, Doha, Qatar.Email:abdelhaleemm@yahoo.de

Amara, A., is an is an assistant professor in Protein Research Department, Genetic Engineering and Biotechnology Research Institute, Mubarak City for Scientific Research and Technology Applications, Alexandria, Egypt. Email: amroamara@hotmail.com

Zaki, S., is an assistant professor in Environmental Biotechnology Department, Genetic Engineering and Biotechnology Research Institute, Mubarak City for Scientific Research and Technology Applications, Alexandria, Egypt. Email: saharzaki@yahoo.com

Abullhamd, A., is an Associate Professor in Microbiology Department, Faculty of Science, Alazhar University, Cairo, Egypt. Email: ashrafabulhamd@yahoo.com

Abulreesh, G., is Ph.D. student in Microbiology Department, Faculty of Science, Alazhar University, Cairo, Egypt and he is an associate researcher at Environmental Biotechnology Department, Genetic Engineering and Biotechnology Research Institute, Mubarak City for Scientific Research and Technology Applications, Alexandria, Egypt. Email: g_abouelrish@yahoo.com

\section{This article should be referenced as follows:}

AbdelHaleem, D.; Amara, A.; Zaki, S.; Abulhamd, A.; Abulreesh, G., (2007). Biosynthesis of biodegradable polyhydroxyalkanotes biopolymers in genetically modified yeasts. Int. J. Environ. Sci. Tech., 4 (4), 513-520. 